\title{
Development of the Ultra-Miniaturized Inertial Measurement Unit WB3 for Objective Skill Analysis and Assessment in Neurosurgery: Preliminary Results
}

\author{
Massimiliano Zecca ${ }^{1,2,3,4}$, Salvatore Sessa ${ }^{2}$, Zhuohua Lin $^{1}$, Takashi Suzuki ${ }^{5}$, \\ Tomoya Sasaki ${ }^{6}$, Kazuko Itoh ${ }^{4,7}$, Hiroshi Iseki ${ }^{5}$, and Atsuo Takanishi ${ }^{1,2,3,4,7,8}$ \\ ${ }^{1}$ Global Robot Academia, Waseda University, Tokyo, Japan \\ zecca@aoni.waseda.jp \\ ${ }^{2}$ Institute for Biomedical Engineering, ASMeW, Waseda University \\ ${ }^{3}$ Humanoid Robotics Institute (HRI), Waseda University \\ ${ }^{4}$ Italy-Japan Joint Laboratory on Humanoid and Personal Robotics "RoboCasa" \\ ${ }^{5}$ FATS, ABMES, Tokyo Women's Medical University, Tokyo, Japan \\ ${ }^{6}$ Graduate School of Science and Engineering, Waseda University \\ ${ }^{7}$ Advanced Research Institute for Science and Engineering, Waseda University \\ ${ }^{8}$ Department of Modern Mechanical Engineering, Waseda University
}

\begin{abstract}
In recent years there has been an ever increasing amount of research and development of technologies and methodologies aimed at improving the safety of advanced surgery. In this context, several training methods and metrics have been proposed, in particular for laparoscopy, both to improve the surgeon's abilities and also to assess her/his skills. For neurosurgery, however, the extremely small movements and sizes involved have prevented until now the development of similar methodologies and systems.

In this paper we present the development of the ultra-miniaturized Inertial Measurement Unit WB3 (at present the smallest, lightest, and best performing in the world) for practical application in neurosurgery as skill assessment tool. This paper presents the feasibility study for quantitative discrimination of movements of experienced surgeons and beginners in a simple pick and place scenario.
\end{abstract}

\section{Introduction}

In recent years, more and more technologies have entered the operating theatre, allowing surgeons to obtain better results and higher performance with great benefits for the patients by using cameras or microscopes and miniaturized tools. While these new technologies have many advantages for patients, they often require surgeons to undergo long and difficult training before achieving the necessary experience. One of the most important issues in this training is the objective evaluation and assessment of surgeon's dexterity and skill, and how they change and evolve over time.

Historically, this kind of evaluation has been subjectively performed by senior surgical staff in both training and operating environments. However it is fundamental to establish more efficient training exercises to enhance the dexterity of surgeons and to define objective metrics for assessing their experience and performance. While several metrics [1-3] and segmentation procedures [4-6] have been employed to characterize 
different phases of surgical movements in laparoscopy, the extremely small movements and sizes involved in neurosurgery (working field is usually $280-370\left[\mathrm{~mm}^{2}\right] \mathrm{x}$ h5-10[cm], target size $0.2-1.5[\mathrm{~mm}]$ [7]) have prevented until now the development of similar methodologies and systems.

Commercial systems such as StealthStation TREONplus by Medtronic or NDI's Aurora Electromagnetic Tracking System require either photoreflecive markers - by which position data are acquired at high frequency; however these markers are heavy and bulky, and they need to be always in sight with the digitizer, which is not always possible [8] - or EM tracking sensors - which are very small, but position data are acquired at relatively low freq, thus limiting the precision of the analysis. One possibility to overcome these limitations and to realize compact measurement systems is nowadays offered by Micro-Electro- Mechanical Systems (MEMS) technology. However, current prototypes such as WB2 [9] and commercial tools such as xSens MTx, InterSense InertiaCube3, and so on, are still too big for a practical application in neurosurgery.

Our aim, therefore, is to develop evaluation tools and to define a set of parameters that allow the characterization of the neurosurgeon's movements during surgery, to see how surgeons of different expertise performs, and to evaluate the improvement of the performance over training. In this paper we present the development of the ultra-miniaturized Inertial Measurement Unit WB3 and its application for the evaluation of surgeon's performance in a simple pick and place scenario.

\section{Material and Methods}

\subsection{WB3 Inertial Measurement Unit IMU}

Our group recently developed a new IMU which is very compact and lightweight (size $20 \times 20$ [mm] and weight 2.9 [g]) - at present the smallest, lightest, and best performing in the world. A picture of the new IMU is shown in Fig. 1. The IMU is composed by the following sensors: 3-axis accelerometer LIS3LV02DL; 2-axis gyroscope IDG300; 1-axis gyroscope LSIY300AL. The IMU's characteristics have been summarized in Table 1. This IMU actually also includes a 3-axis Magnetometer HMC5843; however the data of this sensor have not been analyzed in this paper, and therefore its description is omitted.

The LIS3LV02DL (STMicroelectronics) is a 3-axis accelerometer, whose small size $(4.4 \times 7.5 \times 1[\mathrm{~mm}])$ and high performance characteristics are fully compatible with the strict requirements of neurosurgery. The resolution with a Full-scale $= \pm 2[\mathrm{~g}]$ and Bandwidth $=160[\mathrm{~Hz}]$ is $2[\mathrm{mg}]$, with noise level of about $0.005\left[\mathrm{~m} / \mathrm{sec}^{2}\right]-$ less than one bit. The LISY300AL (STMicroelectronics) is a miniaturized 7.0 x 7.0 x 1.9 [mm] z-axis gyro sensor. Its full-scale is \pm 300 [deg/s] with a Bandwidth of $88[\mathrm{~Hz}]$ and a sensibility of $3.3[\mathrm{mV} / \mathrm{deg} / \mathrm{s}]$. In order to measure 3 -axes angular velocities, we also used a bi-axial gyro IDG300 (InvenSense). The IDG300 size is $6.0 \times 6.0 \times 1.5$ [mm], the measurement range is $\pm 500[$ degree/s] and the sensitivity is $2.0[\mathrm{mV} / \mathrm{deg} / \mathrm{s}]$. Unlike all other prototypes and commercial IMUs available today, this mixed configuration allows our IMU to obtain all the 3 axis of the gyros in one planar layer. 


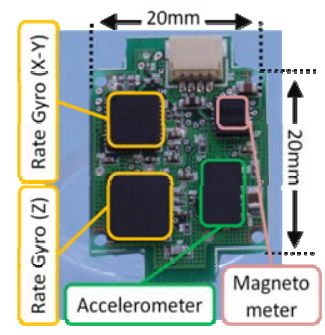

Fig. 1. The new Inertial Measurement Unit WB3

Table 1. Main characteristics of the inertial sensors in our IMU

\begin{tabular}{|l|l|l|l|}
\hline & LIS3LV02DL & \multicolumn{1}{|c|}{ IDG300 } & LSIY300AL \\
\hline Range & $\pm 2[\mathrm{~g}]$ & $\pm 500[\mathrm{deg} / \mathrm{s}]$ & $\pm 300[\mathrm{deg} / \mathrm{s}]$ \\
\hline Sensitivity & $12 \pm 1 \mathrm{bit}$ & $12 \pm 1 \mathrm{bit}$ & $12 \pm 1 \mathrm{bit}$ \\
\hline Bandwidth & $40 \mathrm{~Hz}$ & $140 \mathrm{~Hz}$ & $88 \mathrm{~Hz}$ \\
\hline Sample Rate & $160 \mathrm{~Hz}$ & $500 \mathrm{~Hz}$ & $500 \mathrm{~Hz}$ \\
\hline Linearity & $\pm 2 \%$ & $<1 \%$ & $\pm 0.8 \%$ \\
\hline Noise level & $0.0051 \mathrm{~m} / \mathrm{s}^{2}$ & $0.35[\mathrm{deg} / \mathrm{s}]$ & $0.26[\mathrm{deg} / \mathrm{s}]$ \\
\hline
\end{tabular}

Our IMU also contains a STMicroelectronics 32 bit microcontroller STM32 Cortex for embedded signal elaboration and data exchange. The communication with the module is performed using a CAN BUS at $1[\mathrm{Mb} / \mathrm{s}]$, directly connected with a PC.

\subsection{Experimental Setup}

During neurosurgery, one of the most commonly used instruments is the bipolar forceps (Fig. 2). The main characteristics of the system we used in our experiments are summarized in Table 2. A connector made by acrylonitrile butadiene styrene (ABS) polymer in rapid prototyping for housing our IMU is placed at the proximal end of the bipolar cutting tool (Fig. 2, right). Our IMU's extremely reduced weight and size allows it to be mounted on the bipolar forceps, and to be used during normal tasks without disturbing the surgeon's performance.

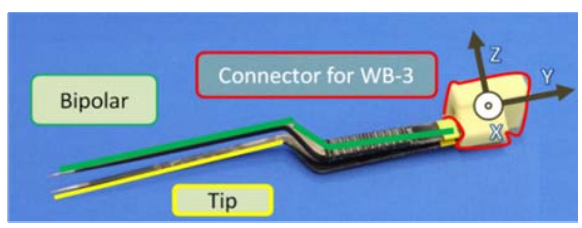

Fig. 2. The Bipolar cutting tool used in our experiments. $\mathrm{X}, \mathrm{Y}$, and $\mathrm{Z}$ axis are indicated
Table 2. Main characteristics of the Bipolar Cutting Tool

\begin{tabular}{|l|c|}
\hline \multicolumn{2}{|c|}{ Bipolar Cutting Tool } \\
\hline Total Length & $194[\mathrm{~mm}]$ \\
\hline Tip Length & $100[\mathrm{~mm}]$ \\
\hline Weight & $34.0[\mathrm{gr}]$ \\
\hline
\end{tabular}

The Skill Evaluation System (SES) used for this preliminary experiment is shown in Fig. 3. SES is composed by 5 main parts:

1) The Testbed, made by ABS, simulates the most common operating space [7]. Current version has a size of $60 \times 40 \times$ h60 [mm].

2) The Support Base (SB) is an aluminium base of $100 \times 100[\mathrm{~mm}]$ with a housing for the testbed made by ABS. SB's purpose is to hold the main unit stable.

3) The Surgical Field (SF, size 50x19 [mm]) simulates the aperture in the human skull. The surgeon accesses the Target Area and the Goal Area from here.

4) The Target Area (TA) is a replaceable soft surface made by Hitohada skin-like gel RTV-2K\#1406 Hardness 0 (EXSEAL Corp., Tokyo, Japan), on which the targets are placed for the experiment. Three different types of TAs, each with 5 small targets randomly placed on it, were prepared to simulate the typical objects that are handled 
during neurosurgery (Fig. 3(b)): BIG: $3.2 \times 1.6$ x 0.4 [mm]; MEDIUM: 2.0 × $1.2 \times 0.3$ [mm]; SMALL: $1.0 \times 0.5 \times 0.2[\mathrm{~mm}]$. Five target areas for each type were prepared in advance to simplify the experimental procedure.

5) The targets picked up from the TA are put in the Goal Area (GA, size $10 \times 30[\mathrm{~mm}]$ ). To facilitate the release of the target, a putty-like adhesive is placed at the bottom of this area.

The experimental setup is shown in Fig. 3(c). The microscope is a MITAKA MRI (Mitaka Kohki Co., Ltd, Tokyo, Japan).
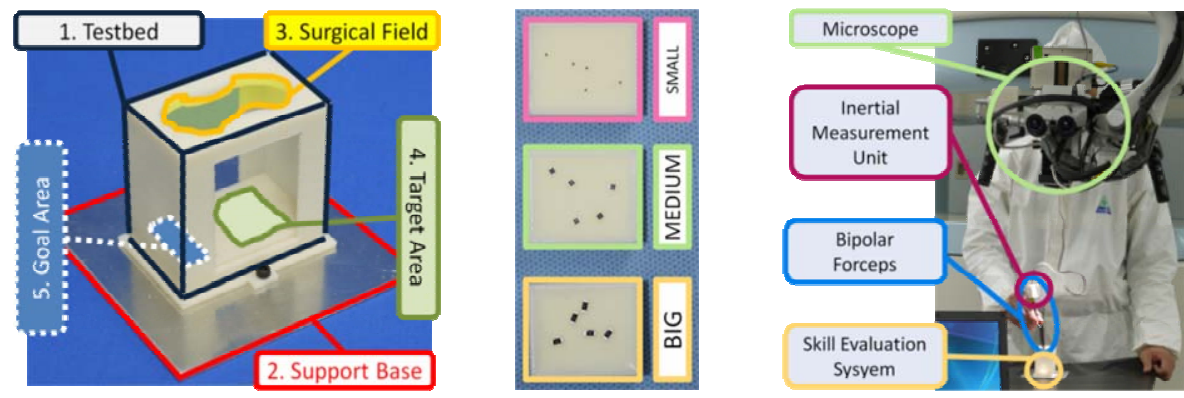

Fig. 3. (a) Picture of the Skill Evaluation System (SES); (b): The 3 types of target area; (c): Overview of the experimental setup

\subsection{Experimental Protocol}

Thirteen non-medical subjects (average age 27.53 years, age range 22-39, all male, all right handed), and 1 professional neurosurgeon (male, age 40, right handed) kindly agreed to participate to the experiments after providing informed consent. Among the non-medical subjects, only 1 had some experience with neurosurgical tools, and 1 had some experience with laparoscopy; all the other subjects were totally novice. The experiments consist in picking all the targets in the target area, one by one, with the bipolar forceps, and releasing them in the goal area. The target area is replaced at the end of each exercise. In total there are 15 target areas ( 3 sizes $x 5$ repetitions), and they are replaced by following the order BIG $\rightarrow$ MEDIUM $\rightarrow$ SMALL, for 5 times.

Acceleration data were sampled at $\mathrm{f}_{\text {acc }}=160[\mathrm{~Hz}]$; gyro data at $\mathrm{f}_{\text {gyro }}=500[\mathrm{~Hz}]$. Data were acquired on the pc for real-time display, and saved for storage and offline analysis. All the data were saved as CSV (Comma Separated Value), and then loaded in Matlab $^{\mathrm{TM}}$ (The MathWorks, Inc.) for further processing and analysis. For each repetition the data were then automatically trimmed to remove dead-time at the beginning and at the end of the trial due to the manual start/stop, as follows: $\mathrm{n}_{\text {start }}$ is defined as the first sample when $\left|a_{y}\right|>9.1 \mathrm{~m} / \mathrm{s}^{2} ; n_{\text {end }}$ is defined as the first sample from the end when $\left|a_{y}\right|>9.1 \mathrm{~m} / \mathrm{s}^{2}$. (Y is the long axis of the bipolar cutting tool as defined in Fig. 2). Only samples between $n_{\text {start }}$ and $n_{\text {end }}$ were then analyzed.

Acceleration components and angular speed components were then filtered and smoothed by using a $10^{\text {th }}$ order bandpass IIR Butterworth filter with cutoff frequencies $\mathrm{f}_{\mathrm{c} 1}=0.05 \mathrm{~Hz}, \mathrm{f}_{\mathrm{c} 2}=8 \mathrm{hz}$ (accelerometers), $\mathrm{f}_{\mathrm{c} 1}=0.05 \mathrm{~Hz}, \mathrm{f}_{\mathrm{c} 2}=5 \mathrm{~Hz}$ (rate gyros), to remove bias and to remove physiological tremor [10]. 
In this paper we present the calculation and analysis of the following variables: Execution Time $\mathrm{T}_{\text {task }}$; Acceleration module $|\mathrm{a}|$; and Angular Speed module $|\omega|$. The fft for $|\mathrm{a}|$ was calculated with $\mathrm{FFT}_{\text {size }}=8192$ samples and frequency resolution $\mathrm{f}_{\text {res }(\mathrm{acc})}=\mathrm{f}_{\text {acc }} / \mathrm{FFT}_{\text {size }}=160 / 8192=0.0195[\mathrm{~Hz}]$. The fft for $|\omega|$ was calculated with frequency resolution $\mathrm{f}_{\text {res }(\text { gyro })}=f_{\text {gyro }} / \mathrm{FFT}_{\text {size }}=0.061[\mathrm{~Hz}]$. The Power Spectral Density PSD was estimated in both cases with the following formula: (fft $\cdot \operatorname{conj}(\mathrm{fft})) / \mathrm{fft}_{\text {size }} \cdot$ The frequency range chosen for the evaluation was $0.2-8[\mathrm{~Hz}]$ for $\mathrm{fft}(|\mathrm{a}|)$, and $0.2-2[\mathrm{~Hz}]$ for $\mathrm{fft}(|\omega|)$, to take into account only the voluntary movements. Several other parameters were also calculated, but are not presented here due to space limitation.

\section{Experimental Evaluation}

The following sections present the details about the experimental evaluation. In the following figures, (norm.) indicates that the data have been normalized to the average corresponding data of the surgeon (Subject \#14) for an easier visual comparison of the scales. The normalizing values are always indicated. Surgeon's data are always displayed with dark gray bars, while novice's data are displayed in light grey. Moreover, the y-scales of the normalized graphs are optimized in function of the surgeon's value, which means that some bar exceeds the y-scale limit (i. e. Fig. 6).

\subsection{Analysis of the Execution Time}

The surgeon - as expected - proved to be always faster (i.e. lower $\mathrm{T}_{\text {task }}$ ) and showed high constancy in the execution time (i.e. lower var $\left(\mathrm{T}_{\text {task }}\right)$ ) of the different tasks than all the novices (Fig. 4). In addition, it can be noticed that both BIG (Fig. 5, left) and MEDIUM (not shown) targets s howed a fast learning effect for the novices, with the execution time stabilizing after the $3^{\text {rd }}$ trial; however, these learning effects could not be seen for the SMALL target (Fig. 5, right). It's also worth noticing that this learning effect is also present with the surgeon for his first trials (not shown).
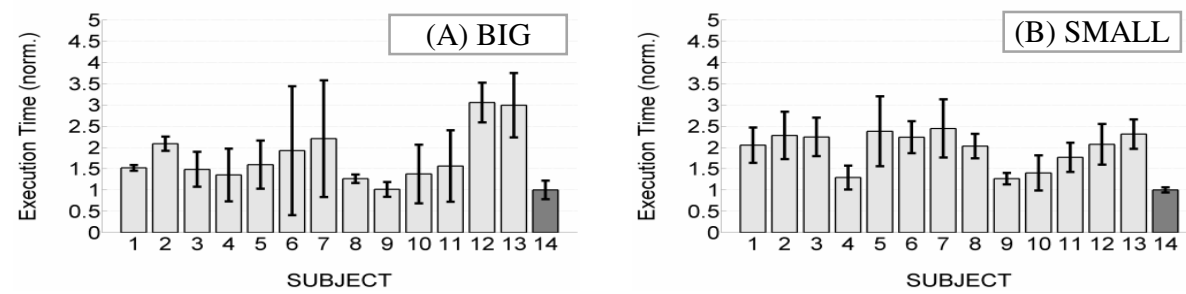

Fig. 4. Execution time for the BIG (left) and for the SMALL (right) targets, averaged on the 5 trials for each subject. Normalization $\mathrm{T}_{\text {task }}$ values are 11.75 and 16.17 [s], respectively

\subsection{Analysis of the Acceleration}

As can be seen in Fig. 6, the mean Power Spectral Density $\overline{\operatorname{PSD}_{|\mathrm{a}|}}$ ) clearly identifies the experienced neurosurgeon (for the surgeon it is 0.62 and $\left.1.47\left[\left(\mathrm{~m} / \mathrm{s}^{2}\right)^{2}\right)\right]$ for the BIG and SMALL task, respectively; while for the other subjects it's usually twice as high or 
more). Frequency distribution of $\operatorname{PSD}_{|\mathrm{a}|}$ is also quite different. It can also be seen that subjects \#2 and \#9 performed very similarly to the neurosurgeon.

Another parameter which shows some differences between the surgeon and the novices is the Cumulative Distribution Function CDF of the acceleration module $|\mathrm{a}|$, calculated as $\operatorname{CDF}(|\mathrm{a}|)=\mathrm{P}(\mathrm{X} \leq|\mathrm{a}|)$ and evaluated for $\mathrm{X}=95 \%\left(\mathrm{CDF}_{95 \%}\right)$. For the BIG targe $\mathrm{t}$ (Fig. 7 (left)) the neurosurgeon outperforms all the novices; the data about the MEDIUM target shows similar trends. Things are different for the SMALL target, when several novices have similar or lower $\mathrm{CDF}_{95 \%}(\mathrm{a} \mid)$ than the neurosurgeon. These however are due to a much higher $\mathrm{T}_{\text {task }}$ for the novices.
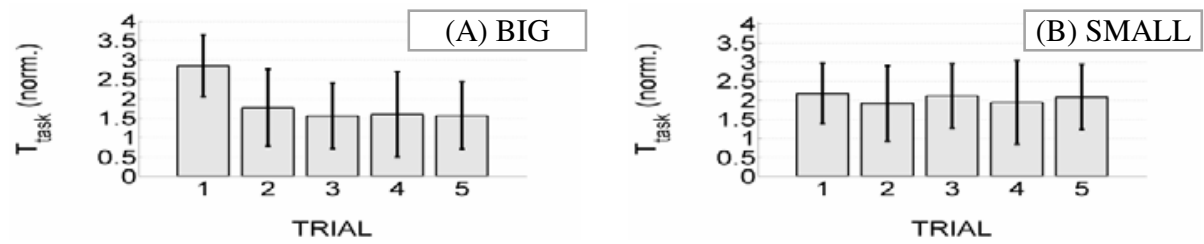

Fig. 5. Execution time for the BIG (left) and for the SMALL (right) targets averaged on all non-medical subjects for each trial. Normalization $\mathrm{T}_{\text {task }}$ are 11.75 and 16.17 [s], respectively.
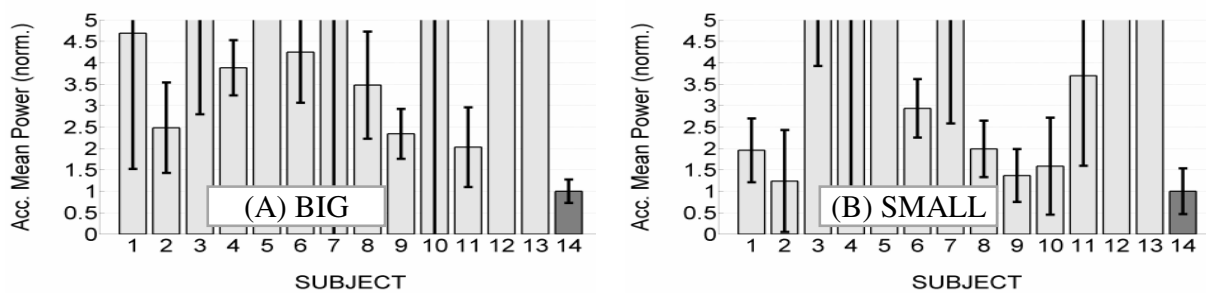

Fig. 6. PSD for the (A) BIG and for the (B) SMALL targets, averaged on the 5 trials for each subject. Normalization values are 0.62 and $\left.1.47\left[\left(\mathrm{~m} / \mathrm{s}^{2}\right)^{2}\right)\right]$.
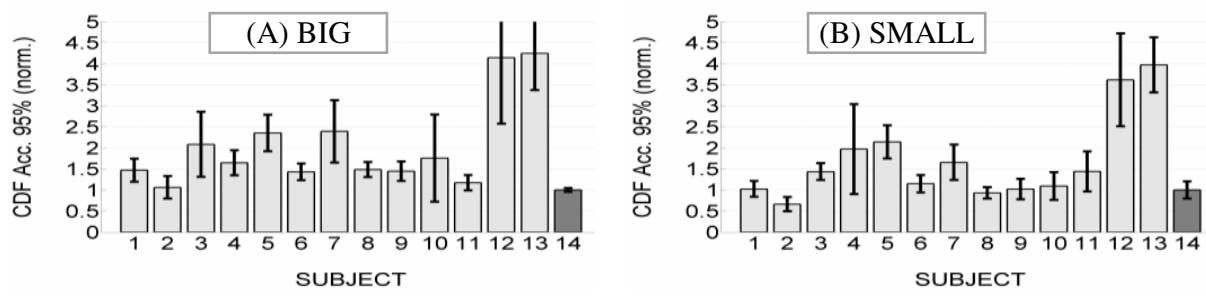

Fig. 7. Cumulative Distribution Function (CDF) of the acceleration $|a|$; for the (A) BIG and (B) SMALL targets. Normalization values are 1.10 and $1.38\left[\mathrm{~m} / \mathrm{s}^{2}\right]$, respectively.

\subsection{Analysis of the Rate Gyro}

Among the different parameters, the Cumulative Distribution Function (CDF) of the angular speed $\operatorname{CDF}(|\omega|)=P(X \leq|\omega|)$ evaluated for $X=95 \%\left(C_{95 \%}\right)$ shows some 

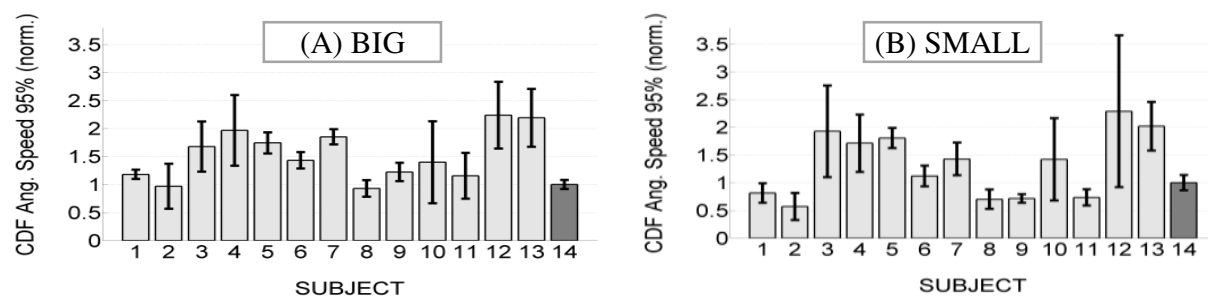

Fig. 8. CDF of the angular speed. Normalization values are 16.6 and 19.3 [deg/s].

difference between the surgeon and the n ovices (Fig. 8). As with $\operatorname{CDF}_{95 \%}(|a|)$, , the surgeon's $\mathrm{CDF}_{95 \%}(|\omega|)$ is usually lower than the other subjects for the BIGGER and MEDIUM targets, showing therefore high smoothness in movement; it's intermediate for the SMALL targets, where some subject reached a lower $\operatorname{CDF}_{95 \%}(|\omega|)$ at the expenses of a higher $\mathrm{T}_{\text {task }}$. $\mathrm{CDF}_{95 \%}(|\omega|)$ also shows a very limited variance for the surgeon, thus signifying high regularity in the exercises.

\section{Discussion and Conclusions}

With the diffusion of more and more advanced tools and technologies in the operating room, it is fundamental to establish more efficient training exercises and to define objective metrics to objectively evaluate the dexterity of neurosurgeons. The extremely small movements and sizes involved in neurosurgery, however, have prevented until now the development of such methodologies and systems. In this paper we presented the development of an ultra-miniaturized Inertial Measurement Unit WB3 (Sec. 2.A). The extreme lightweight and its extreme high performance make WB3 suitable for applications in neurosurgery. Moreover, it directly acquires accelerations and rotations at high frequency, thus providing smooth and virtually noise-free data. Commercial systems, instead, acquire target's position and then obtain rotations and accelerations by successive derivations which introduce more noise.

As a preliminary test we applied WB3 to a bipolar forceps (Sec. 2), the most commonly used instrument in neurosurgery, and we used this system to evaluate the movements of neurosurgeons in a simple pick and place scenario. The preliminary results (Sec. 3) proved that several parameters extracted from the IMU's data (and in particular the mean Power Spectral Density $\overline{\operatorname{PSD}_{|\mathrm{a}|}}$ and the Cumulative Distribution Function $\mathrm{CDF}_{95 \%}$ of both acceleration $|\mathrm{a}|$ and angular speed $|\omega|$ ) allow a clear distinction between a professional neurosurgeon and a group of novices; moreover, these data also could show which non-medical subject performs similarly to the surgeon, and how, thus validating the approach proposed in this pilot study.

In the future, the data of more professional subjects, as well as the data of both more non-medical and more medical novice subjects are needed to define a more precise evaluation system. Overall, this work shows that a substantial set of parameters is necessary to investigate and analyze the performance of surgeons. Currently, work is still in progress, and our future commitment in this field is to continue to analyze the performance of neurosurgeons in more complex exercises and procedures. 
Acknowledgments. This research has been conducted at the Waseda University Center for Advanced Biomedical Sciences/TWIns. Partial support was also provided by: ASMeW Priority Research C Grant \#11; JSPS Grant-in-aid for Scientific Research \#19700389 and \#20700386; JSPS Postdoctoral Fellowship Program for Foreigner Researcher FY2008; G-COE Global Robot Academia; Advancement of University Education Project of Chinese government; Waseda Grant for Special Research Projects \#266740. The authors would like to express their thanks to the Italian Ministry of Foreign Affairs, General Directorate for Cultural Promotion and Cooperation, for its support to RoboCasa. The authors would also like to express their gratitude to STMicroelectronics, Dyden Corp, Japan ROBOTECH LTD, SolidWorks Corp, Okino Industries LTD, for their support to the research.

\section{References}

1. Cotin, S., Stylopoulos, N., Ottensmeyer, M.P., Neumann, P., Rattner, D.W., Dawson, S.L.: Metrics for laparoscopic skills trainers: The weakest link! In: Dohi, T., Kikinis, R. (eds.) MICCAI 2002. LNCS, vol. 2488, pp. 35-43. Springer, Heidelberg (2002)

2. Verner, L., Oleynikov, D., Holtmann, S., Haider, H., Zhukov, L.: Measurements of the level of surgical expertise using flight path analysis from da VinciTM robotic surgical system. Medicine Meets Virtual Reality 11, 373-378 (2003)

3. Stylopoulos, N., Vosburgh, K.G.: Assessing technical skill in surgery and endoscopy: a set of metrics and an algorithm to assess skills in surgical and endoscopic procedures. Surg. Innov. 14, 113-121 (2007)

4. Risucci, D., Cohen, J.A., Garbus, J.E., Goldstein, M., Cohen, M.G.: The effects of practice and instruction on speed and accuracy during resident acquisition of simulated laparoscopic skills. Curr. Surg. 58, 230-235 (2001)

5. Payandeh, S., Lomax, A., Dill, J., Mackenzie, C., Cao, C.G.L.: On Defining Metrics for Assessing Laparoscopic Surgical Skills in a Training Envirnoment. Studies in Health Technology and Informatics - Medicine Meets Virtual Reality, vol. 85, pp. 334-340. IOS Press, Amsterdam (2002)

6. Megali, G., Sinigaglia, S., Tonet, O., Dario, P.: Modelling and evaluation of surgical performance using hidden Markov models. IEEE transactions on bio-medical engineering 53, 1911-1919 (2006)

7. Gonzalez, L.F., Crawford, N.R., Horgan, M.A., Deshmukh, P., Zabramski, J.M., Spetzler, R.F.: Working area and angle of attack in three cranial base approaches: pterional, orbitozygomatic, and maxillary extension of the orbitozygomatic approach. Neurosurgery 50 , 550-555; discussion 555-557 (2002)

8. Woerdeman, P.A., Willems, P.W., Noordmans, H.J., van der Sprenkel, J.W.: The analysis of intraoperative neurosurgical instrument movement using a navigation log-file. Int. J. Med. Robot. 2, 139-145 (2006)

9. Zecca, M., Saito, M., Endo, N., Mizoguchi, Y., Itoh, K., Takanobu, H., Takanishi, A.: Development of the Waseda Bioinstrumentation System WB-2 - the new Inertial Measurement Unit. In: IEEE International Conference on Robotics and Biomimetics (Robio 2007), Sanya, China, pp. 139-144 (2007)

10. Ibanez, D.O., Baquerin, F.P., Choi, D.Y., Riviere, C.N.: Performance Envelope and Physiological Tremor in Microsurgery. In: Proceedings of the IEEE 32nd Annual Bioengineering Conference, pp. 121-122 (2006) 